\title{
Novel Bioelectric Mechanisms and Functional Significance of Peripheral and Central Entrainment by Respiration
}

\author{
Ravinder Jerath*, Connor Beveridge \\ Charitable Medical Healthcare Foundation, Augusta, GA, USA \\ Email: ${ }^{*}$ Rj605r@aol.com
}

How to cite this paper: Jerath, R. and Beveridge, C. (2018) Novel Bioelectric Mechanisms and Functional Significance of Peripheral and Central Entrainment by Respiration. World Journal of Neuroscience, 8, 480-500.

https://doi.org/10.4236/wjns.2018.84038

Received: November 2, 2018

Accepted: November 25, 2018

Published: November 28, 2018

Copyright ( 92018 by authors and Scientific Research Publishing Inc. This work is licensed under the Creative Commons Attribution International License (CC BY 4.0).

http://creativecommons.org/licenses/by/4.0/

\begin{abstract}
The human organism is a complex biological system with emergent properties that arise from the unified functional interactions among its diverse components. When studying the brain and body in light of modern biological systems approaches, one must analyze them in a holistic manner, putting aside reductionist models in order to understand how certain properties manifest from complex system interactions. The respiratory system is capable of continuously adapting to changes in the internal and external environment, making it one of the most integrated of physiological processes. We propose an additional respiratory process: respiration-derived electrical currents during inspiration that spread throughout the entire body maintaining homeostasis through entraining oscillatory activity, modulating cognitive processes, and modulating the autonomic nervous system. If these currents are indeed created in part from redox reactions occurring on a massive scale, then we assert they are a major aspect of an embodied cognitive framework. We propose that this potentially major source of organism integrity has been overlooked, and its application to medicine could drastically change how we understand human physiology, the autonomic nervous system, and the therapeutic treatment of various clinical disorders.
\end{abstract}

\section{Keywords}

Bioelectric, Respiration, Embodied Cognition, Oxidation, Membrane Potential, Neural Oscillation, Hyperpolarization

\section{Introduction}

The heart-lung machine was first used on humans in 1953, modernizing coronary surgery by giving doctors significantly more time to operate on the heart 
[1]. This machine bypasses the lungs and heart, oxygenating the blood and pumping it. This technique brings into question whether the lungs or heart is truly needed to survive. However, it has become clear that this technique is not such an impeccable replacement to the cardiopulmonary system as it has resulted in what is known among surgeons as "pumphead" [2]. This syndrome, post perfusion syndrome, is characterized by short and long-term cognitive decline and includes defects in attention, concentration, memory, motor function, and response time [3]. We make a case for the existence of an additional source of required homeostatic energy that may explain this syndrome and a plethora of physiological phenomena. The source of energy we explore is electrical in nature. We propose that an immediate release of electrons via redox reactions occurs during inspiration, as well as hyperpolarizing cells throughout the body and brain, followed by depolarization during expiration. In the case of the "pumphead", we assert the time spent without cardiopulmonary activity which resulted in the patient's deprival of a vital electrical, global entrainment that is provided during respiration. These electrical currents are vital in that they underlie an oscillatory cognitive architecture than can be deconstructed when the energy is absent.

The vital electrical currents we describe are unknown to modern science with unknown properties which could be studied from a variety of angles. Research into the formal discovery of this energy and why it may be necessary for cellular and cognitive vitality will likely reveal the origin of the cognitive deficits underlying post perfusion syndrome. We propose that starving cells of this vital homeostatic energy could lead to dysfunction among cellular assemblies and the resulting poor cognitive outcomes of cardiopulmonary bypass [4]. Additionally, a better understanding of these currents will likely reveal the nature of the variety of respiration-induced neural oscillations that are present throughout the brain and body. In this article, we not only explore the effect respiration that has on neuro-physiological processes, but also biochemical mechanisms that may provide this electrical energy.

Models of embodied cognitive science, which include psychological and biological systems, consider the brain, mind, and body as a single entity. Although this scientific field is not unified in its established definitions [5], its main thesis is that the body not only affects the mind, but the mind affects the body via dynamic interactions [6]; for example, holding a warm cup of coffee versus a cold one leads participants to judge someone as more trustworthy after a brief interaction [7]. Respiration has not received much attention in the embodied cognition literature, and we aim to elucidate its psychological and physiological role in various aspects of the mechanisms of mind. Failing to include such bodily aspects will lead to incomplete cognitive models.

\section{Prevailing Knowledge of Respiration Physiology}

The current state of scientific understanding in systems physiology is incomplete 
with undiscovered functions of organs being revealed even recently. For instance, the lungs have recently been revealed to play a significant and unexpected role in the production of blood [8]. Current scientific understanding in the field of cardiopulmonology describes how breathing brings oxygen to alveoli in the lungs which through diffusion exchange oxygen for carbon dioxide in the blood [9]. These gases are transported to and from cells throughout the body via the circulatory system [10]. Respiratory and cardiovascular systems are tightly coupled in the brainstem where respiratory rhythms have been proposed to be generated [11]. The exact mechanisms of these regular rhythms remain under debate [12]. These continuous central breathing patterns are generated involuntarily and they adapt rapidly to changing physiological, psychological, and environmental conditions [13]. Breathing may be the most integrated physiological process with various metabolic and behavioral processes modulating its function including sleep, arousal, fear, exercise, and hypercapnia. There is also a close interaction between breathing and higher brain functioning reflected in how it can modulate the mind resulting in fear, arousal, and cognitive changes [14]. Although many neuro-cellular components of breathing have been well researched, how these components integrate with the much larger respiratory network remains poorly understood. Through an introduction to components of the respiratory network, we will be able to describe effectively the role this energy plays in its global function.

\subsection{The Pre-Bötzinger Complex}

The Pre-Bötzinger complex is a neuronal group in the brainstem that is proposed to be the main factor in generating respiratory rhythms [15]. Anatomical alterations of this complex have been shown to associate with distinct breathing abnormalities [16]. It is considered the central pattern generator, driving motor activities during inspiration [13]. Rythmogenesis by this complex is modulated by membrane properties and synaptic interactions and can therefore be regulated by neurotransmitters and electrical activity [14]. Although many studies confirm the importance of this complex in various aspects of breathing [17] [18] [19] [20], it is part of a larger network that is distributed through the nervous system [14].

\subsection{Cardiovascular Coupling and Modulation}

The extensive integration of the respiratory network includes the cardiovascular system. There are often various types of coupling between heart activity and respiration, however, the clinical applications of these phenomena are poorly understood [21]. One of these integrative phenomena is a change in heart rate according to the current phase of the respiratory cycle known as respiratory sinus arrhythmia (RSA) [22], with heart rate quickening during inspiration and slowing during expiration [23]. Furthermore, cardiorespiratory phase synchronization and cardiorespiratory coordination can be observed during various mental 
and physiological states [21]. Phase synchronization is a coupling in phase while coordination is a coupling in time [21]. While these various couplings often occur simultaneously, they are fundamentally different. Synchronization is found during times of relaxation, while coordination is found in the opposite state of stress [21]. Heart rate variability of RSA is maximized at around six breaths per minute, indicating cardiorespiratory resonance [24].

The physiological significance of RSA is still mysterious; however, such significance is demonstrated to likely exist. The efficiency of pulmonary gas exchange has been shown to be improved through RSA, likely by replacing pulmonary capillary blood with each heartbeat [23]. Heart rate is determined by the firing frequency of a group of electrically active pacemaker cells in the right atrium known as the sinoatrial node [25]. The frequency of this node is determined by the balance of sympathetic and parasympathetic activity relayed to it with this activity being modulated in part by respiration in addition to the brainstem which contains projections from the cortex and limbic system [23]. During inspiration, cardiac parasympathetic neurons are hyperpolarized, and activity of the cardiac vagal nerve is nearly abolished leading to the inspiratory tachycardia of RSA [23]. During expiration, this activity is maximized leading to bradycardia [23]. Heart rate variability can be used as an index of a cardiovascular health [22], and atypical RSA patterns have been identified with higher levels of depression and other illness [26].

In addition to effects on cardiac rhythms, respiration has also been shown to modulate blood pressure and vascular resistance. Blood pressure has been shown to correlate with respiratory rhythms [27]. It increases with inspiration and falls during the expiratory process [28]. Cerebral oxyhemoglobin concentration fluctuations overlap and correlate with frequencies of the respiratory rhythm in response to breathing patterns [29] [30].

\subsection{Hering Breuer Reflex and Pulmonary Stretch Receptors}

Slowly Adapting Stretch receptors of the lungs, discovered in 1868 by Hering and Breuer, are mechanoreceptors that respond to lung inflation [31]. These receptors have been evidenced to be involved in modulating breathing pattern [32], vascular resistance, heart rate [31], and autonomic tone [33]. Although these mechanisms are not fully understood [31], they are demonstrated to prevent excessive lung inflation, facilitating expiration via hyperpolarizing inputs to the brain stem in a process known as the Hering Breuer reflex [32]. The inhibitory action potentials travel along the vagus nerve to "pump" cells in the medullary region of the brainstem [34] which then inhibit inspiratory neurons involved in respiratory pattern and rhythm generation including the ventral respiratory column and the Pre-Bötzinger complex [32].

\subsection{Autonomic Activity}

The autonomic nervous system (ANS) is the major neural network involved in 
respiration, playing a major role in fits interaction with circulation that was first described over eighty years ago via oscillatory discharge of the sympathetic network synchronizing with respiratory rhythm [23]. However, mechanisms of ANS modulation by respiration remain incomplete. Strong correlations have been made between respiratory patterns and autonomic activity. Faster, more irregular respiration rates are associated with sympathetic tone [35], while slow, deep breathing has been associated with parasympathetic [36] [37] [38]. Although modulation of the ANS has regarded as purely involuntary, through voluntary modulation of respiration, it has been demonstrated that the ANS can be voluntarily influenced [35]. Long-term parasympathetic dominance can also be achieved through slow deep breathing practice over extended periods [39], and parasympathetic activity levels are correlated with depression and the success of techniques that attenuate sadness and dysphoria [26]. In addition, slow deep breathing may allow for optimal sympathovagal balance and improved autonomic reactivity to stress due to augmented baroreflex sensitivity [38]. The baroreflex is a homeostatic mechanism in which baroreceptor neurons in various vascular tissues relay blood pressure information from stretching of vessels to the brainstem [40].

We have discussed in the previous section the role the efferent, cardiac vagal nerve plays in RSA, however there is a deeper relationship between the ANS and respiration. The reflex mechanisms and stretch receptor activities we described converge at the central respiratory centers of the brainstem which also control tones of the ANS [38]. Suppression of sympathetic input by increasing tidal volume during inspiration suggests that slowly adapting stretch receptor activity is a major link between respiration and the ANS.

\section{Effects of Respiration on Behavior and Cognition}

Although capturing oxygen and expelling carbon dioxide is the most important physiological function of breathing, it has many mysterious effects throughout the body and mind that cannot be explained through gas exchange. These interesting effects on various aspects of conscious and unconscious phenomena will be explored here in order to support our hypothesis on a novel respiratory mechanism that transmits electrical currents throughout the body. In particular, many of these instantaneous modulations seem to be differentiated by inspiration and expiration in line with our hypothesis that the body harvests electrons during the massive inflow of oxygen during inspiration, and that these electrons disperse throughout the body causing various effects on the mind and body.

Cognitive science has largely supposed that cognition is a function of brain activity alone; however, a radical new theory of cognition includes various aspects of the body as components of an organism's cognitive architecture. The cognitive science of embodied cognition, which we touched upon in the introduction, considers the brain and body as a unified entity in producing the mind, with the dynamic interactions occurring between these previously presumed, 
distinct systems [6]. Many studies have demonstrated how respiration affects behavior and cognitive activities with some theories on the mechanisms of these effects being proposed. Through our hypothesis, we aim to further embodied cognition science by explaining a physiological mechanism for the instantaneous effects respiration has cognition, thus providing biological foundations for psychological observations related to breathing.

\subsection{Emotions}

Emotions have been thoroughly linked to breathing [41], ANS activity [42], cardiorespiratory coupling, and other physiological changes throughout the body [43]. Respiratory activity is not only determined by interactions among the brainstem and cortical structures, but also the limbic system (specifically the amygdala) [43], which is a functional correlate of emotion [44]. Respiratory patterns can be changed dramatically by emotions [43]. For instance, anxiety can significantly increase expiratory time [45]. When anxiety increases the respiration rate, activity synchronized with respiration can be observed in the limbic system [46]. Voluntarily modulating breathing pattern to those patterns associated with certain emotions may trigger neurological and physiological mechanisms triggered by the corresponding emotions [43]. As an integral part of yogic and breathing exercise, conscious modulation of breathing pattern or focused attention on spontaneous breathing can allow one to change their emotional state [47] [48]. Certain members of the military use such conscious breathing techniques to regain composure during stressful combat situations [49].

Several limbic and paralimbic areas are involved in breathing changes associated with emotional processing [50], and amygdala activity is synchronized with each breath [51]. Detection of emotion via facial expressions occurs more quickly during inspiration compared to expiration, suggesting that synchrony in the amygdala is dependent upon respiratory phase [52]. Irregular patterns of respiration are associated with more stressed states of mind, demonstrated by studies revealing these patterns in patients with panic disorder [53]. Although much research has been conducted on correlations and mechanisms behind the respiration-emotion connection, there has been little discussion on the functional purposes behind why such a connection exists. A major hypothesis was proposed that the connection fulfills response requirements of the emotional situation [41].

\subsection{Perceptions}

Influence by respiration on sensory perception has in part been substantiated through studies on visual and auditory signal detection, revealing that the threshold for signal detection of hard to perceive stimuli is higher during inspiration compared to expiration [54]. During controlled breathing, reaction times are significantly longer during expiration compared to inspiration [55]. Natural, involuntary breathing showed similar extension of reaction time during expiration 
when the detection tasks are more complex [52]. Pain is experienced as less severe during expiration compared to inspiration [56], and focused, slow breathing in general has been shown to reduce pain [57].

\subsection{Motor Function}

A basic function of motor control, the force generated upon muscle contraction, has been significantly correlated with the respiratory cycle. Handgrip force is notably stronger during forced expiration than during forced inspiration [58]. Eye movements have been demonstrated to be phase locked to respiration [59]. Motion tracking precision and response latency with finger movements has also been shown to be differentiated during inspiration and expiration [60]. These effects may occur via respiratory modulation of neural communications between the motor cortex and motor neurons in the spinal cord [61].

\subsection{Cognition}

As part of an embodied cognitive architecture, respiration should show direct influence on cognitive processes. This has been shown in studies with variations during inspiration and expiration. Memory retrieval has been shown to be more accurate when the memories are accessed during inspiration compared to expiration [52]. Neuronal oscillations in the gamma range are correlated with a wide variety of cognitive activities such as attention [62], problem solving [63], and decision making [64]. Findings that respiration can modulate gamma oscillations in the neocortex suggest that respiration can modulate cognition [65].

\subsection{Entrainment of Brain Rhythms}

Although conscious control and awareness of breathing has been used therapeutically since prehistoric times [66], the mechanisms underlying its efficacy remains poorly understood. Although neuronal oscillations have been known to lock to breathing cycles in the olfactory bulb since the 1940's [67], recent findings have begun to suggest that breathing can act as a global organizer of neuronal oscillations throughout the human brain [52] [68]. By modulating intrinsic gamma network oscillations, respiration may organize cortical excitability [69]. These entrainments have been observed in multiple brain regions, however, they are most prominent in the frontal lobe [70] (Figure 1). Gamma-respiration coupling is dominant during awake states while it is diminished during REM sleep when theta coupling increases [71]. The lack of recognition of these oscillatory influences may have negatively altered results of previous studies on oscillatory coupling and cross-regional synchrony in the brain as the authors did not recognize certain rhythms appearing due to respiration [70].

The respiration entrainment of neural networks has been proposed to contribute to information processing [72], information transfer [71], and linking distributed cell assemblies [70]. The possibility of these entrainments being due to mechanical artifacts has been voided by the demonstration that the entrainments 

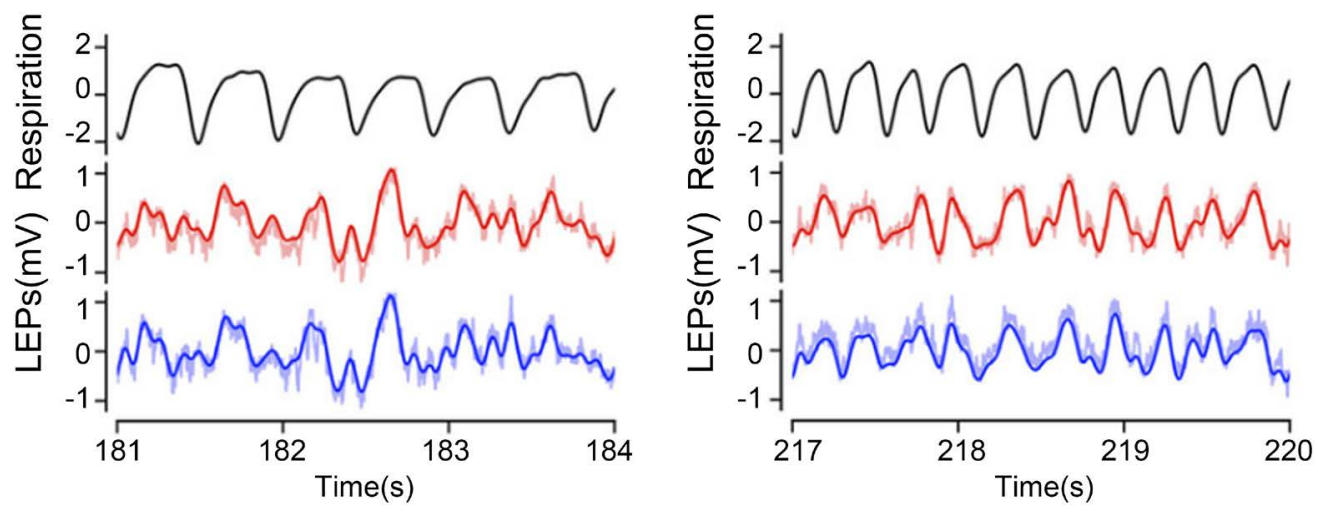

Figure 1. Entrainment of neural oscillations by respiration. This chart contains local field potential data from the somatosensory cortex of the awake mouse and demonstrates neural entrainment by respiration. Similar data has been obtained in human subjects [65] (Copyright permissions obtained from Copyright Clearance Center (C).

are specific to grey matter versus white matter or cerebrospinal fluid [68]. Therefore, these neuronal entrainments must have a physiologic function and we propose they are part of an embodied cognitive architecture influencing conscious processes. Oscillatory activity in the brain is hierarchically organized such that higher frequency oscillations are modulated by lower frequencies [73]. Low frequency oscillations such as respiratory rhythms tend to be coherent over large distances and well suited for interregional communication [74]. Therefore, respiration rhythms may provide a foundation for the distributed, more locally functioning higher neural frequencies to organize upon [68]. These insights may provide mechanisms involved in therapeutic breathing exercises.

\section{Membrane Potential Homeostasis as a Fundamental Structure of Cognition}

Many metastable consciousness models such as the Default Space Theory and the Operational Architectonics theory assert that consciousness arises from a global bioelectric structure arising from micro to macro level, synchronous, neural oscillations [75]. The macro oscillations arise from synchronized firing of action potentials with subthreshold membrane potential oscillations occurring at the micro level [76]. Without sufficient membrane hyperpolarization above the action potential threshold, excessive and chaotic action potential firing would occur leading to excitotoxicity [77] and therefore disruption of cognitive processes including consciousness [78] [79]. The harmful effects of excessive depolarization can be observed in stroke and cerebral ischemia which include neuronal dysfunction and death [80]. Conditions of excessive neural excitation include epilepsy [81], autism [82], and Parkinson's disease [83].

Excessive hyperpolarization can also result in homeostatic catastrophe. Over-hyperpolarization of respiratory neurons by certain drugs can prevent respiration and lead to death [84]. In the healthy person, excitation-to-inhibition ratio remains constant resulting in about an equal number of excitatory and in- 
hibitory neurons [85]. Over inhibition of neurons has also been associated with schizophrenia [82]. These observations in part form our assertion that the maintenance of membrane potential is the most basic requirement of higher cognitive processes. In order to maintain the ion gradients producing the membrane potential, ATPase pumps force $\mathrm{Na}^{+}$and $\mathrm{K}^{+}$across their electrochemical gradients. This continuous process requires a steady supply of energy in the form of ATP (adenosine triphosphate). Leaky ion channels prevent these pumps from over-hyperpolarizing the membranes [86]. Neurons also have been shown to increase excitatory or inhibitory synapses as a homeostatic response to over inhibition or excitation, respectively [85]. In addition to ATP powered membrane polarization and homeostatic mechanisms, we propose an additional mechanism for maintaining healthy membrane potential of all cells.

\section{Hypothesis}

We have reviewed the prevailing knowledge of respiratory physiology in relation to neural and bodily physiologic systems in order to provide the basis for a novel hypothesis. The hypothesis we are proposing will elucidate a secondary function of respiration, however, this mechanism is of major importance to the majority of the members of the Animalia kingdom by coordinating vital homeostatic rhythms and providing a source of energy for cellular functions. We propose that via the dynamic mechanical activity of stretch receptors in the lungs and redox reactions that occur primarily at the site of gas exchange, electrical currents are produced that are used throughout the entire body. The vast numbers of electrons of these currents are derived from the oxygen we exchange per breath which numbers at $6 \times 10^{23}$ molecules of $\mathrm{O}_{2}$. Because these specific redox reactions and activity we describe occur heavily at the site of gas exchange in the alveoli, their instantaneous effect on the body and mind is contrasted with the many seconds in takes for blood to travel around the body, thus providing an explanation for the instantaneous effects breathing can have on the body and brain. The cellular energy these currents provide contrast the intracellular energy production occurring in the mitochondria in that it is an extracellular source delivered to the cells. We propose that these bioelectric currents provide not only a source of cellular energy, but also maintain homeostasis, modulate cognitive processes, create a central pattern generator for respiration, and modulate the autonomic nervous system through entrainment of oscillatory activity throughout the body.

\subsection{Sources of Electrical Current}

The main source of the electrical current we are proposing generated during respiration arises via common redox reactions, which release electrons from the oxygen molecules being brought into the lungs during inspiration. In addition, we propose more speculative means of current generation to be researched including currents by the mechanical stretching of slowly-adapting stretch recep- 
tors as well as when carbon dioxide is exchanged into the volume of air in the lungs. During inspiration, these electrons produce hyperpolarization in cell membranes throughout the body by traveling throughout bodily tissues leading to the effects on the body, perception, and cognition we have discussed (Figure 2). During expiration, the cellular membranes are globally depolarized, likely stimulating respiratory drive [87]. It is by this mechanism that respiration plays a fundamental role in an embodied cognitive architecture. These sources of electrical current are described below.

We propound that the electrons released via these sources we shall describe hyperpolarize cells throughout the body not through free electrons accumulating within cells, but via the acceptance of these electrons by intracellular proteins and acids. Membrane impermeable organic acids and proteins exist at a greater concentration within the cell and balance the intracellular positive charge held by the large amount potassium ions within when they possess a negative charge [88]. According to our hypothesis, membrane-permeable electrons become situated within the cell by being accepted by these internal acids and proteins which cannot diffuse out of the cell. These become anions which act to hyperpolarize the cell. As anions they are the main source of negative charge within the cell of a resting membrane and a major component of the cytoplasm.

Recent evidence suggests that organisms may harness some features of quantum mechanical effects for physiological use [89]. We suggest in light of modern research in quantum biology, the transport of electrons throughout the body
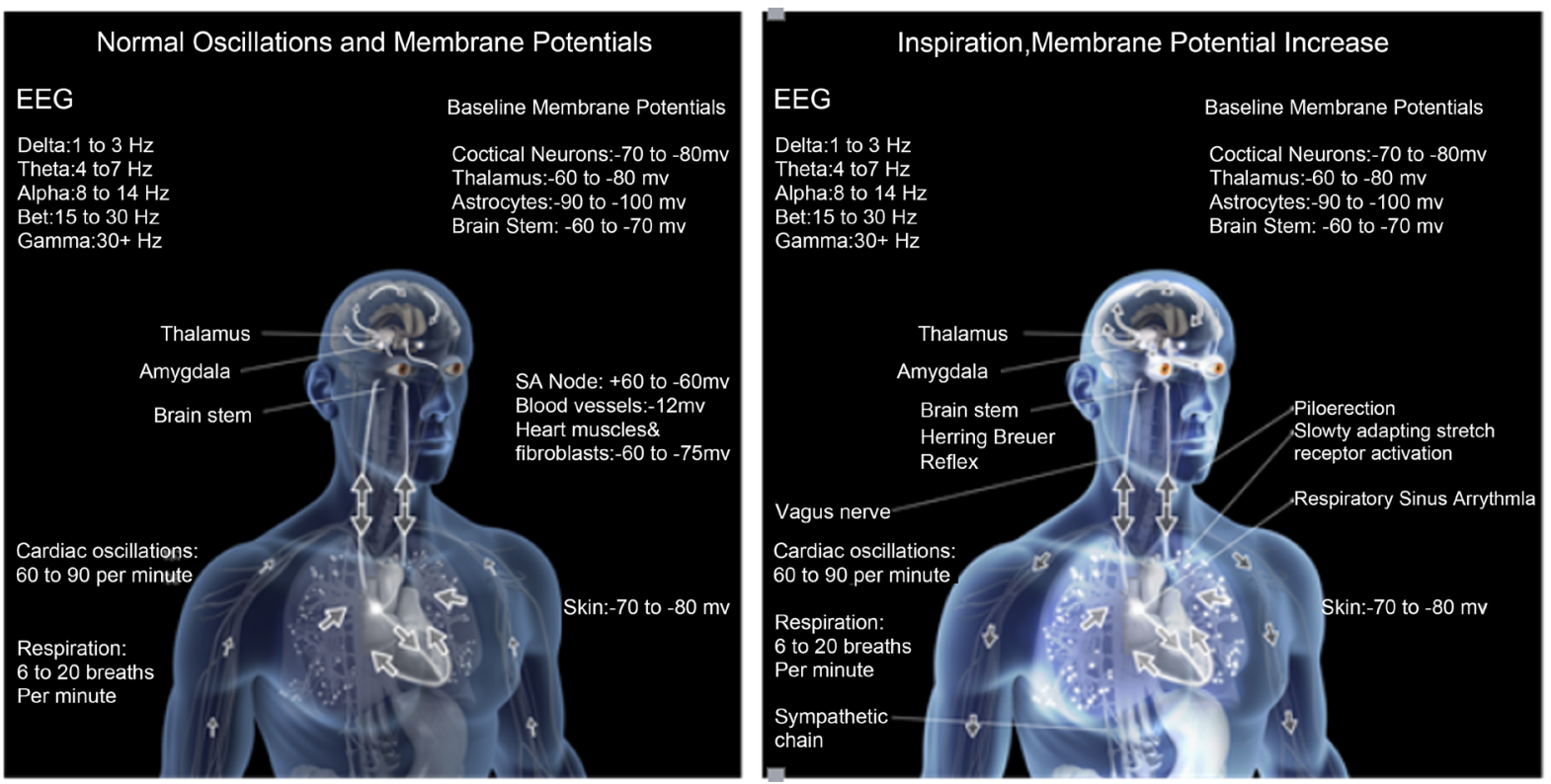

Figure 2. Inspiration effects on global membrane potential. This figure illustrates the electrical activity that occurs during inspiration. During inspiration, electrical currents distributed by the sources we describe cause global hyperpolarization in all the body's cells, illustrated here by white illumination. Via these currents, instantaneous physiological changes take place: respiratory sinus arrhythmia in the heart, the Herring Breuer Reflex in the brain stem, hyperpolarization and inhibition of sympathetic neurons, and entrainment of neural oscillations that may play a role in global organization of oscillatory activity [93] (Permission obtained by SCIRP Publishing). 
may be assisted by quantum tunneling. Quantum tunneling is the ability of small particles such as electrons to pass through energy barriers [90]. Enzymes have been demonstrated to use quantum tunneling to transport electrons long distances (tens of angstroms) between redox centers during cellular respiration [89] [91] [92]. Via quantum tunneling, we believe electrons harvested from inspiration may be transferred across energy barriers during the redox reactions we have described.

\subsubsection{Source \#1: Redox Reactions in Lung Parenchyma}

Aerobic respiration occurs in all cells of the body using oxygen to create ATP [94]. Although this occurs in all cells of the body, there exists a much greater oxygen concentration in the lung parenchyma during inspiration. The lung parenchyma consists of numerous thin-walled alveoli which create an immense surface area allowing for effective gas exchange [95]. Each alveolus is surrounded by numerous capillaries at which gases are exchanged by diffusion [96]. We propose a source of electrical current that is part of the ATP synthesis process which occurs on a timescale of tens of milliseconds [97]. ATP formation from $\mathrm{ADP}$ and phosphate is an energetically unfavorable process [98]. In the endothelial cells of the capillaries and epithelial cells of the alveoli, glycolysis and the citric acid cycle take place with exposure to very high concentrations of oxygen. The products of this process are then used in the mitochondria for the electron transport chain which has the function of producing a transmembrane proton electrochemical gradient as the result of redox reactions [99]. The electron transport chain consists of a series of redox reactions which transfer electrons from donors to acceptors. We propose that electrons from redox reactions of this chain escape the cycle and are transferred to tissues throughout the body as electrical current. Because the oxygen concentration in the lung parenchyma is so much more dense than in the rest of the body, a much greater number of electrons are released there leading to the flow of current away from the lungs.

\subsubsection{Source \#2: Hemoglobin and Myoglobin Oxygenation and 0xidation} When oxygen diffuses into the blood at the alveolar capillaries, it is bound to the heme group of myoglobin and hemoglobin [100]. When these heme groups are oxygenated, they are oxidized, meaning they lose electrons [101]. It is this oxidation we propose as a source of electrical current (Equation (1)). Although some of these electrons are transferred to oxygen forming super oxide, studies that trace the electrons once they are removed from the ferrous cation of the heme are absent. We assert they are distributed globally through transmission among all tissues of the body and brain.

$$
\mathrm{Hem}-\mathrm{Fe}^{2+}+\mathrm{O}_{2}=\mathrm{Hem}-\mathrm{Fe}^{3+}-\mathrm{O}_{2}+\mathrm{e}^{-}
$$

\subsubsection{Source \#3: Hemoglobin Release of Carbon Dioxide}

Just as red blood cells accept oxygen at the alveolar capillaries, they also release carbon dioxide in the process of gas exchange. We assert another source of elec- 
trical current produced at the site of gas exchange. When hemoglobin carries carbon dioxide, it is referred to as carbaminohemoglobin [102]. When carbaminohemoglobin arrives at the lungs, the binding of incoming oxygen displaces the carbon dioxide along with some protons [103]. We adduce that the release of each proton coincides with the release of an electron. The leads to a significant amount of electron release as up to 3 moles per day of carbon dioxide is released via the carbaminohemoglobin pathway [103].

\subsubsection{Source \#4: Mechanical Generation of Current by Slowly Adapting Stretch Receptors}

The mechanosensory activity of stretch receptors in the lungs occurs when the volume of the lungs expand [104] (Figure 3). Although highly speculative, we have formed a conjecture that the mechanical energy from stretching receptors is converted via a biological mechanism into electrical energy. We propose that via such physical mechanisms, slowly adapting stretch receptors are another source of electrical current. These inhibitory currents we describe may inhibit the respiratory center upon sufficient lung inflation leading to deflation, thus providing mechanisms for the central pattern generation for respiration. In addition, rhythms transmitted to the brainstem via action potentials may influence activity elsewhere.
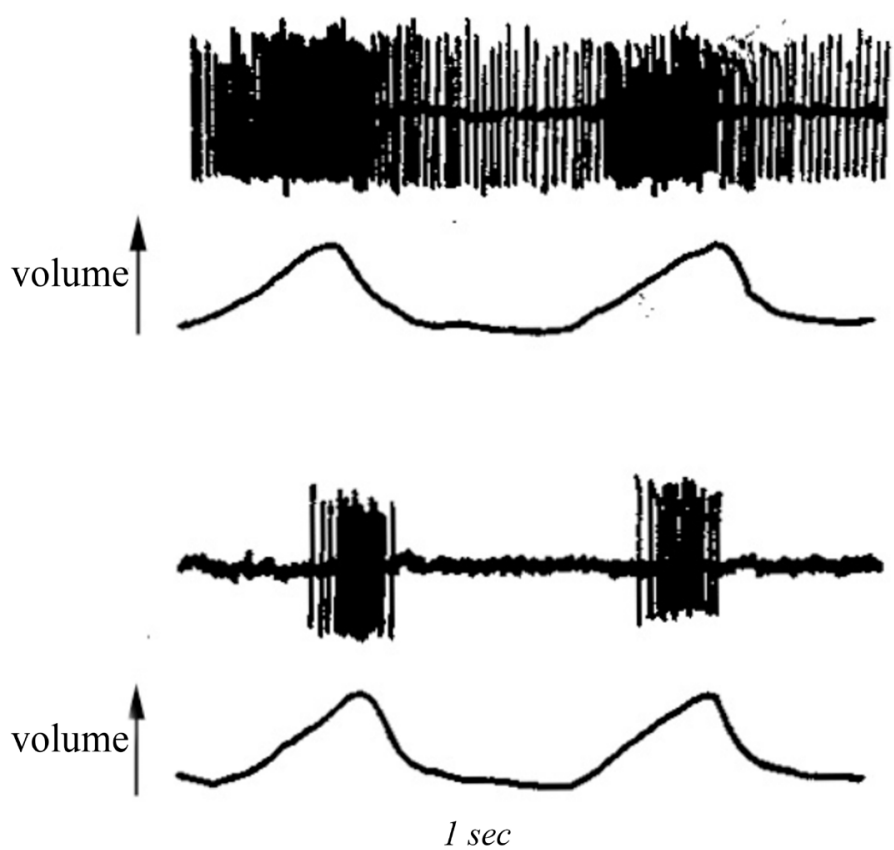

Figure 3. Activity of slowly activated stretch receptors. This image documents the activity of slowly activated stretch receptors when lung expansion exceeds end-expiratory level. Different types of these receptors activate in different patterns as shown; the top image shows a receptor type which stays active during expiration while the bottom image shows a receptor which only activates during inspiration. When expiration occurs, the activity softens or ceases. In addition to the activity scene, we propose that these receptors generate electrical activity that is distributed throughout the body. Image from Mortola [105]. 


\subsection{A Central Pattern Generator}

In addition to the bodily and cognitive modulations by these respiratory electrical currents we have proposed, we assert they play a central role in the maintenance of the respiratory pattern itself, thus being part of its own central pattern generator. This pattern occurs in a cyclical manner with each phase being a phase of respiration, beginning with the harvesting of electrons from the sources discussed (Figure 4). The electrical currents that spread throughout the body then cause a slight hyperpolarization of all cells. This inhibition along with inhibitory currents from the stretch receptors influences the respiratory center strong enough to cause the Pre-Bötzinger complex to cease its signals to the motor activities driving inspiration; thus, the mechanism of the Hering Breuer reflex is extended by our hypothesis beyond the stretch receptors. These inhibitory currents also inhibit the sympathetic nervous system and increase vagal tone. We propound that through global hyperpolarization and then depolarization among expiration, they produce the respiratory rhythms that have been identified in the brain, heart, and vascular tissues.

Once inspiration ceases primarily due to the inhibition to respiratory centers

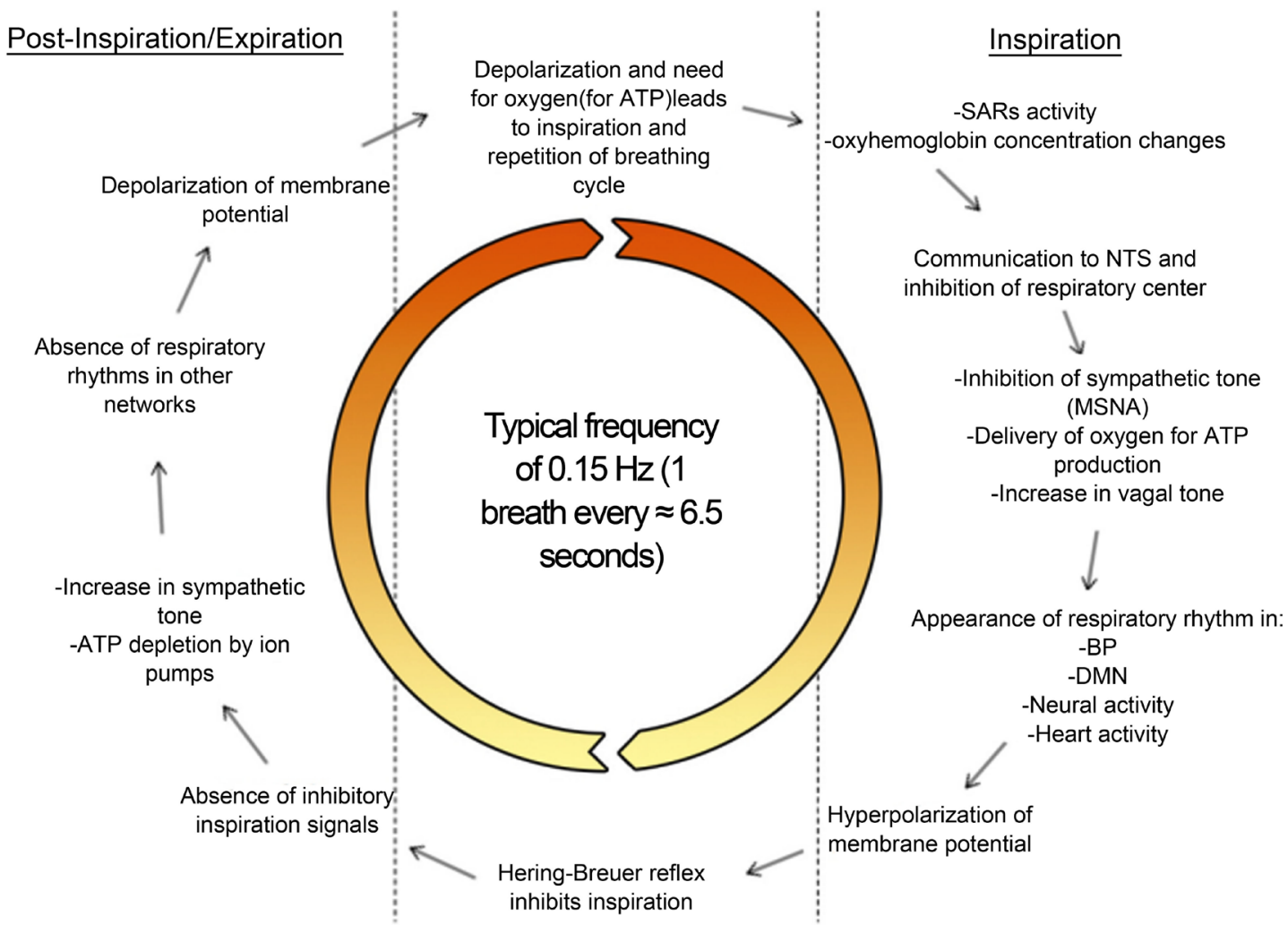

Figure 4. Respiration generated electrical currents as a central pattern generator. This chart illustrates how respiration not only influences the body and membrane potential, but produces respiratory drive when it is needed. Inhibitory currents produced upon inspiration affect the ANS, respiratory systems in the brainstem, and rhythms throughout the body via hyperpolarization. Upon expiration, the currents cease and depolarization occurs, further affecting rhythms throughout the body and stimulating respiratory drive. Figure adapted from Jerath et al., 2015 [87]. 
in the brainstem, the diaphragm relaxes and due to the elasticity of the lungs, the natural composure of the chest wall and abdominal structures compress the lungs in a recoil effect [106]. Upon expiration, the global hyperpolarization of cells begins to reverse as a mechanism of homeostasis. This depolarization causes the stimulation of the sympathetic nervous system, as well as having effects on global oscillatory rhythms. The global depolarization then stimulates respiratory drive, initiating inspiration and restarting the respiratory cycle.

\section{Conclusion}

We have proposed a novel mechanism which can explain the various entrainments on various bodily and neural activities by respiration. Electrical currents generated by redox reactions and mechanical activity at the site of the lungs may modify these activities via the global hyperpolarization of cellular membranes. The sources of electrical current via the release of electrons include oxygen rich redox reactions in the lung parenchyma through the electron transport chain, redox reactions in the alveolar capillaries via heme groups, the release of carbon dioxide at the site of gas exchange, and the potential mechanical generation by slowly adapting stretch receptors in the lungs. Studies will need to be conducted to validate this novel hypothesis. Studies may begin with monitoring electrical currents in cells throughout the body in phase with respiration. No discussion of this hypothesis has been published besides our own work. This hypothesis will help to explain how different patterns of breathing have various, and instantaneous effects on the body and mind. Through these sources of energy, the incomplete theories of respiratory patterns which include the Pre-Bötzinger complex and the Hering Breuer reflex can be made whole. This proposition will also help explain and form a more complete understanding of human physiology. Finally, by describing physiological phenomena for theories of embodied cognition, more accurate models of how cognition occurs not only in the brain, but also through the body can be developed.

\section{Acknowledgements}

We would like to thank Dr. Vernon Barnes for editing the manuscript. Funding has been provided by Charitable Medical Healthcare Foundation.

\section{Author's Contributions}

Theory developed by RJ with some writing with majority of the manuscript written by $\mathrm{CB}$.

\section{Funding}

Funding Provided by Charitable Medical Healthcare Foundation.

\section{Conflicts of Interest}

The authors declare no conflict of interest. 


\section{References}

[1] Cohn, L.H. (2003) Fifty Years of Open-Heart Surgery. Circulation, 107, 2168-2170. https://doi.org/10.1161/01.CIR.0000071746.50876.E2

[2] Alston, R.P. (2005) Pumphead-Or Not! Does Avoiding Cardiopulmonary Bypass for Coronary Artery Bypass Surgery Result in Less Brain Damage? BJA: British Journal of Anaesthesia, 94, 699-701. https://doi.org/10.1093/bja/aei128

[3] Jensen, B.O., Hughes, P., Rasmussen, L.S., Pedersen, P.U. and Steinbrüchel, D.A. (2006) Cognitive Outcomes in Elderly High-Risk Patients after Off-Pump versus Conventional Coronary Artery Bypass Grafting. A Randomized Trial. Circulation, 113, 2790-2795. https://doi.org/10.1161/CIRCULATIONAHA.105.587931

[4] Parolari, A., Alamanni, F., Cannata, A., Naliato, M., Bonati, L., Rubini, P., Veglia, F., Tremoli, E. and Biglioli, P. (2003) Off-Pump versus On-Pump Coronary Artery Bypass: Meta-Analysis of Currently Available Randomized Trials. The Annals of Thoracic Surgery, 76, 37-40. https://doi.org/10.1016/S0003-4975(03)00183-8

[5] Wilson, M. (2002) Six Views of Embodied Cognition. Psychonomic Bulletin \& Review, 9, 625-636. https://doi.org/10.3758/BF03196322

[6] Wilson, R.A. and Foglia, L. (2017) Embodied Cognition. In: The Stanford Encyclopedia of Philosophy, Spring 2017 Edition Edition, Metaphysics Research Lab, Stanford University, Stanford.

[7] Williams, L.E. and Bargh, J.A. (2008) Experiencing Physical Warmth Promotes Interpersonal Warmth. Science, 322, 606-607. https://doi.org/10.1126/science.1162548

[8] Lefrançais, E., Ortiz-Muñoz, G., Caudrillier, A., Mallavia, B., Liu, F., Sayah, D.M., Thornton, E.E., Headley, M.B., David, T., Coughlin, S.R., Krummel, M.F., Leavitt, A.D., Passegué, E. and Looney, M.R. (2017) The Lung Is a Site of Platelet Biogenesis and a Reservoir for Haematopoietic Progenitors. Nature, 544, 105-109. https://doi.org/10.1038/nature21706

[9] Hall, J. (2011) Guyton and Hall Textbook of Medical Physiology. Saunders/Elsevier, Philadelphia.

[10] Pocock, G. and Richards, C.D. (2006) Human Physiology: The Basis of Medicine. Oxford University Press, Oxford.

[11] Abdala, A.P.L., Rybak, I.A., Smith, J.C., Zoccal, D.B., Machado, B.H., St-John, W.M. and Paton, J.F.R. (2009) Multiple Pontomedullary Mechanisms of Respiratory Rhythmogenesis. Respiratory Physiology \& Neurobiology, 168, 19-25.

https://doi.org/10.1016/j.resp.2009.06.011

[12] Guerrier, C., Hayes, J.A., Fortin, G. and Holcman, D. (2015) Robust Network Oscillations during Mammalian Respiratory Rhythm Generation Driven by Synaptic Dynamics. Proceedings of the National Academy of Sciences of the United States of America, 112, 9728-9733. https://doi.org/10.1073/pnas.1421997112

[13] Kam, K., Worrell, J.W., Janczewski, W.A., Cui, Y. and Feldman, J.L. (2013) Distinct Inspiratory Rhythm and Pattern Generating Mechanisms in the Prebötzinger Complex. The Journal of Neuroscience, 33, 9235-9245. https://doi.org/10.1523/JNEUROSCI.4143-12.2013

[14] Ramirez, J., Doi, A., Garcia, A., Elsen, F., Koch, H. and Wei, A. (2012) The Cellular Building Blocks of Breathing. In: Terjung, R., Ed., Comprehensive Physiology.

[15] Smith, J.C., Ellenberger, H.H., Ballanyi, K., Richter, D.W. and Feldman, J.L. (1991) Pre-Bötzinger Complex: A Brainstem Region That May Generate Respiratory Rhythm in Mammals. Science, 254, 726-729. 
https://doi.org/10.1126/science.1683005

[16] Schwarzacher, S.W., Rub, U. and Deller, T. (2010) Neuroanatomical Characteristics of the Human Pre-Botzinger Complex and Its Involvement in Neurode-Generative Brainstem Diseases. Brain, 134, 24-35. https://doi.org/10.1093/brain/awq327

[17] Thoby-Brisson, M., Trinh, J.-B., Champagnat, J. and Fortin, G. (2005) Emergence of the Pre-Bötzinger Respiratory Rhythm Generator in the Mouse Embryo. The Journal of Neuroscience, 25, 4307-4318. https://doi.org/10.1523/JNEUROSCI.0551-05.2005

[18] Mckay, L.C. and Feldman, J.L. (2008) Unilateral Ablation of Pre-Bötzinger Complex Disrupts Breathing during Sleep but Not Wakefulness. American Journal of Respiratory and Critical Care Medicine, 178, 89-95. https://doi.org/10.1164/rccm.200712-19010C

[19] Tan, W., Pagliardini, S., Yang, P., Janczewski, W.A. and Feldman, J.L. (2010) Projections of PreBötzinger Complex Neurons in Adult Rats. The Journal of Comparative Neurology, 518, 1862-1878. https://doi.org/10.1002/cne.22308

[20] Ramirez, J.-M. (2011) The Human Pre-Bötzinger Complex Identified. Brain, 134, 8-10. https://doi.org/10.1093/brain/awq357

[21] Krause, H., Kraemer, J.F., Penzel, T., Kurths, J. and Wessel, N. (2017) On the Difference of Cardiorespiratory Synchronisation and Coordination. Chaos: An Interdisciplinary Journal of Nonlinear Science, 27, Article ID: 093933. https://doi.org/10.1063/1.4999352

[22] Elstad, M., O'callaghan, E.L., Smith, A.J., Ben-Tal, A. and Ramchandra, R. (2018) Cardiorespiratory Interactions in Humans and Animals: Rhythms for Life. American Journal of Physiology Heart and Circulatory Physiology, 315, H6-H17. https://doi.org/10.1152/ajpheart.00701.2017

[23] Yasuma, F. and Hayano, J. (2004) Respiratory Sinus Arrhythmia: Why Does the Heartbeat Synchronize with Respiratory Rhythm? Chest, 125, 683-690. https://doi.org/10.1378/chest.125.2.683

[24] Vaschillo, E.G., Vaschillo, B. and Lehrer, P.M. (2006) Characteristics of Resonance in Heart Rate Variability Stimulated by Biofeedback. Applied Psychophysiology and Biofeedback, 31, 129. https://doi.org/10.1007/s10484-006-9009-3

[25] Monfredi, O., Dobrzynski, H., Mondal, T., Boyett, M.R. and Morris, G.M. (2010) The Anatomy and Physiology of the Sinoatrial Node-A Contemporary Review. Pacing and Clinical Electrophysiology, 33, 1392-1406. https://doi.org/10.1111/j.1540-8159.2010.02838.x

[26] Yaroslavsky, I., Rottenberg, J., Bylsma, L.M., Jennings, J.R., George, C., Baji, I., Benák, I., Dochnal, R., Halas, K., Kapornai, K., Kiss, E., Makai, A., Varga, H., Vetró, Á. and Kovacs, M. (2016) Parasympathetic Nervous System Activity Predicts Mood Repair Use and Its Effectiveness among Adolescents with and without Histories of Major Depression. Journal of Abnormal Psychology, 125, 323-336. https://doi.org/10.1037/abn0000149

[27] Bernardi, L., Radaelli, A., Solda, P.L., Coats, A.J., Reeder, M., Calciati, A., Garrard, C.S. and Sleight, P. (1996) Autonomic Control of Skin Microvessels: Assessment by Power Spectrum of Photoplethysmographic Waves. Clinical Science, 90, 345-355. https://doi.org/10.1042/cs0900345

[28] Hanson, B., Gill, J., Western, D., Gilbey, M.P., Bostock, J., Boyett, M.R., Zhang, H., Coronel, R. and Taggart, P. (2012) Cyclical Modulation of Human Ventricular Repolarization by Respiration. Frontiers in Physiology, 3, 379.

https://doi.org/10.3389/fphys.2012.00379 
[29] Katura, T., Tanaka, N., Obata, A., Sato, H. and Maki, A. (2006) Quantitative Evaluation of Interrelations between Spontaneous Low-Frequency Oscillations in Cerebral Hemodynamics and Systemic Cardiovascular Dynamics. Neuroimage, 31, 1592-1600. https://doi.org/10.1016/j.neuroimage.2006.02.010

[30] Pfurtscheller, G., Daly, I., Bauernfeind, G. and Muller-Putz, G.R. (2012) Coupling between Intrinsic Prefrontal $\mathrm{HbO}_{2}$ and Central EEG Beta Power Oscillations in the Resting Brain. PLoS ONE, 7, e43640. https://doi.org/10.1371/journal.pone.0043640

[31] Schelegle, E.S. (2003) Functional Morphology and Physiology of Slowly Adapting Pulmonary Stretch Receptors. The Anatomical Record Part A Discoveries in Molecular Cellular and Evolutionary Biology, 270, 11-16. https://doi.org/10.1002/ar.a.10004

[32] Kubin, L., Alheid, G.F., Zuperku, E.J. and Mccrimmon, D.R. (2006) Central Pathways of Pulmonary and Lower Airway Vagal Afferents. Journal of Applied Physiology, 101, 618-627. https://doi.org/10.1152/japplphysiol.00252.2006

[33] Goso, Y., Asanoi, H., Ishise, H., Kameyama, T., Hirai, T., Nozawa, T., Takashima, S., Umeno, K. and Inoue, H. (2001) Respiratory Modulation of Muscle Sympathetic Nerve Activity in Patients with Chronic Heart Failure. Circulation, 104, 418-423. https://doi.org/10.1161/hc2901.093111

[34] Miyazaki, M., Arata, A., Tanaka, I. and Ezure, K. (1998) Activity of Rat Pump Neurons Is Modulated with Central Respiratory Rhythm. Neuroscience Letters, 249, 61-64. https://doi.org/10.1016/S0304-3940(98)00402-9

[35] Kox, M., Van Eijk, L.T., Zwaag, J., Van Den Wildenberg, J., Sweep, F.C.G.J., Van Der Hoeven, J.G. and Pickkers, P. (2014) Voluntary Activation of the Sympathetic Nervous System and Attenuation of the Innate Immune Response in Humans. Proceedings of the National Academy of Sciences, 111, 7379-7384. https://doi.org/10.1073/pnas.1322174111

[36] Jerath, R., Edry, J.W., Barnes, V.A. and Jerath, V. (2006) Physiology of Long Pranayamic Breathing: Neural Respiratory Elements May Provide a Mechanism That Explains How Slow Deep Breathing Shifts the Autonomic Nervous System. Medical Hypotheses, 67, 566-571. https://doi.org/10.1016/j.mehy.2006.02.042

[37] Chang, Q., Liu, R. and Shen, Z. (2013) Effects of Slow Breathing Rate on Blood Pressure and Heart Rate Variabilities. International Journal of Cardiology, 169, e6-e8. https://doi.org/10.1016/j.ijcard.2013.08.121

[38] Russo, M.A., Santarelli, D.M. and O'rourke, D. (2017) The Physiological Effects of Slow Breathing in the Healthy Human. Breathe, 13, 298-309. https://doi.org/10.1183/20734735.009817

[39] Pal, G.K., Velkumary, S. and Madanmohan (2004) Effect of Short-Term Practice of Breathing Exercises on Autonomic Functions in Normal Human Volunteers. Indian Journal of Medical Research, 120, 115-121.

[40] Eckberg, D.L. and Orshan, C.R. (1977) Respiratory and Baroreceptor Reflex Interactions in Man. Journal of Clinical Investigation, 59, 780-785. https://doi.org/10.1172/JCI108699

[41] Boiten, F.A., Frijda, N.H. and Wientjes, C.J.E. (1994) Emotions and Respiratory Patterns: Review and Critical Analysis. International Journal of Psychophysiology, 17, 103-128. https://doi.org/10.1016/0167-8760(94)90027-2

[42] Davis, M. (1992) The Role of the Amygdala in Fear and Anxiety. Annual Review of Neuroscience, 15, 353-375. https://doi.org/10.1146/annurev.ne.15.030192.002033

[43] Homma, I. and Masaoka, Y. (2008) Breathing Rhythms and Emotions. Experimen- 
tal Physiology, 93, 1011-1021. https://doi.org/10.1113/expphysiol.2008.042424

[44] Pessoa, L. (2010) Emotion and Cognition and the Amygdala: From "What Is It?" to “What's to Be Done?" Neuropsychologia, 48, 3416-3429. https://doi.org/10.1016/j.neuropsychologia.2010.06.038

[45] Masaoka, Y. and Homma, I. (1999) Expiratory Time Determined by Individual Anxiety Levels in Humans. Journal of Applied Physiology, 86, 1329-1336. https://doi.org/10.1152/jappl.1999.86.4.1329

[46] Masaoka, Y. and Homma, I. (2000) The Source Generator of Respiratory-Related Anxiety Potential in the Human Brain. Neuroscience Letters, 283, 21-24. https://doi.org/10.1016/S0304-3940(00)00895-8

[47] Brown, R.P. and Gerbarg, P.L. (2005) Sudarshan Kriya Yogic Breathing in the Treatment of Stress, Anxiety, and Depression: Part I-Neurophysiologic Model. The Journal of Alternative and Complementary Medicine, 11, 189-201. https://doi.org/10.1089/acm.2005.11.189

[48] Arch, J.J. and Craske, M.G. (2006) Mechanisms of Mindfulness: Emotion Regulation Following a Focused Breathing Induction. Behaviour Research and Therapy, 44, 1849-1858. https://doi.org/10.1016/j.brat.2005.12.007

[49] Grossman, D. and Christensen, L.W. (2011) On Combat: The Psychology and Physiology of Deadly Conflict in War and Peace. Human Factor Research Group Inc., Millstadt.

[50] Masaoka, Y., Sugiyama, H., Katayama, A., Kashiwagi, M. and Homma, I. (2012) Remembering the Past with Slow Breathing Associated with Activity in the Parahippocampus and Amygdala. Neuroscience Letters, 521, 98-103. https://doi.org/10.1016/j.neulet.2012.05.047

[51] Masaoka, Y., Izumizaki, M. and Homma, I. (2014) Chapter 19-Where Is the Rhythm Generator for Emotional Breathing? In: Holstege, G., Beers, C.M. and Subramanian, H.H., Eds., Progress in Brain Research, Elsevier, Amsterdam, 367-377.

[52] Zelano, C., Jiang, H., Zhou, G., Arora, N., Schuele, S., Rosenow, J. and Gottfried, J.A. (2016) Nasal Respiration Entrains Human Limbic Oscillations and Modulates Cognitive Function. The Journal of Neuroscience, 36, 12448-12467. https://doi.org/10.1523/JNEUROSCI.2586-16.2016

[53] Abelson, J.L., Weg, J.G., Nesse, R.M. and Curtis, G.C. (2001) Persistent Respiratory Irregularity in Patients with Panic Disorder. Biological Psychiatry, 49, 588-595. https://doi.org/10.1016/S0006-3223(00)01078-7

[54] Flexman, J.E., Demaree, R.G. and Simpson, D.D. (1974) Respiratory Phase and Visual Signal Detection. Perception \& Psychophysics, 16, 337-339. https://doi.org/10.3758/BF03203952

[55] Li, S., Park, W. and Borg, A. (2012) Phase-Dependent Respiratory-Motor Interactions in Reaction Time Tasks During Rhythmic Voluntary Breathing. Motor Control, 16, 493-505. https://doi.org/10.1123/mcj.16.4.493

[56] Iwabe, T., Ozaki, I. and Hashizume, A. (2014) The Respiratory Cycle Modulates Brain Potentials, Sympathetic Activity, and Subjective Pain Sensation Induced by Noxious Stimulation. Neuroscience Research, 84, 47-59. https://doi.org/10.1016/j.neures.2014.03.003

[57] Zautra, A.J., Fasman, R., Davis, M.C. and Craig, A.D. (2010) The Effects of Slow Breathing on Affective Responses to Pain Stimuli: An Experimental Study. PAIN, 149, 12-18. https://doi.org/10.1016/j.pain.2009.10.001

[58] Li, S. and Laskin, J.J. (2006) Influences of Ventilation on Maximal Isometric Force 
of the Finger Flexors. Muscle \& Nerve, 34, 651-655.

https://doi.org/10.1002/mus.20592

[59] Rassler, B. and Raabe, J. (2003) Co-Ordination of Breathing with Rhythmic Head and Eye Movements and with Passive Turnings of the Body. European Journal of Applied Physiology, 90, 125-130. https://doi.org/10.1007/s00421-003-0876-5

[60] Rassler, B. (2000) Mutual Nervous Influences between Breathing and Precision Finger Movements. European Journal of Applied Physiology, 81, 479-485. https://doi.org/10.1007/s004210050071

[61] Varga, S. and Heck, D.H. (2017) Rhythms of the Body, Rhythms of the Brain: Respiration, Neural Oscillations, and Embodied Cognition. Consciousness and Cognition, 56, 77-90. https://doi.org/10.1016/j.concog.2017.09.008

[62] Tallon-Baudry, C. (2004) Attention and Awareness in Synchrony. Trends in Cognitive Sciences, 8, 523-525. https://doi.org/10.1016/j.tics.2004.10.008

[63] Sheth, B.R., Sandkühler, S. and Bhattacharya, J. (2009) Posterior Beta and Anterior Gamma Oscillations Predict Cognitive Insight. Journal of Cognitive Neuroscience, 21, 1269-1279. https://doi.org/10.1162/jocn.2009.21069

[64] Siegel, M., Engel, A. and Donner, T. (2011) Cortical Network Dynamics of Perceptual Decision-Making in the Human Brain. Frontiers in Human Neuroscience, 5. https://doi.org/10.3389/fnhum.2011.00021

[65] Ito, J., Roy, S., Liu, Y., Cao, Y., Fletcher, M., Lu, L., Boughter, J.D., Grün, S. and Heck, D.H. (2014) Whisker Barrel Cortex Delta Oscillations and Gamma Power in the Awake Mouse Are Linked to Respiration. Nature Communications, 5, 3572. https://doi.org/10.1038/ncomms4572

[66] Everly, G. and Lating, J. (2002) A Clinical Guide to the Treatment of Human Stress Response.

[67] Adrian, E.D. (1942) Olfactory Reactions in the Brain of the Hedgehog. The Journal of Physiology, 100, 459-473. https://doi.org/10.1113/jphysiol.1942.sp003955

[68] Herrero, J.L., Khuvis, S., Yeagle, E., Cerf, M. and Mehta, A.D. (2018) Breathing above the Brain Stem: Volitional Control and Attentional Modulation in Humans. Journal of Neurophysiology, 119, 145-159. https://doi.org/10.1152/jn.00551.2017

[69] Heck, D.H., Mcafee, S.S., Liu, Y., Babajani-Feremi, A., Rezaie, R., Freeman, W.J., Wheless, J.W., Papanicolaou, A.C., Ruszinkó, M., Sokolov, Y. and Kozma, R. (2017) Breathing as a Fundamental Rhythm of Brain Function. Frontiers in Neural Circuits, 10. https://doi.org/10.3389/fncir.2016.00115

[70] Tort, A.B.L., Brankačk, J. and Draguhn, A. (2018) Respiration-Entrained Brain Rhythms Are Global But Often Overlooked. Trends in Neurosciences, 41, 186-197. https://doi.org/10.1016/j.tins.2018.01.007

[71] Zhong, W., Ciatipis, M., Wolfenstetter, T., Jessberger, J., Müller, C., Ponsel, S., Yanovsky, Y., Brankačk, J., Tort, A.B.L. and Draguhn, A. (2017) Selective Entrainment of Gamma Subbands by Different Slow Network Oscillations. Proceedings of the National Academy of Sciences of the United States of America, 114, 4519-4524. https://doi.org/10.1073/pnas.1617249114

[72] Biskamp, J., Bartos, M. and Sauer, J.-F. (2017) Organization of Prefrontal Network Activity by Respiration-Related Oscillations. Scientific Reports, 7, Article No. 45508. https://doi.org/10.1038/srep45508

[73] Lakatos, P., Shah, A.S., Knuth, K.H., Ulbert, I., Karmos, G. and Schroeder, C.E. (2005) An Oscillatory Hierarchy Controlling Neuronal Excitability and Stimulus Processing in the Auditory Cortex. Journal of Neurophysiology, 94, 1904-1911. 
https://doi.org/10.1152/jn.00263.2005

[74] Hyafil, A., Giraud, A.-L., Fontolan, L. and Gutkin, B. (2015) Neural Cross-Frequency Coupling: Connecting Architectures, Mechanisms, and Functions. Trends in Neurosciences, 38, 725-740. https://doi.org/10.1016/j.tins.2015.09.001

[75] Jerath, R. and Beveridge, C. (2018) Top Mysteries of the Mind: Insights from the Default Space Model of Consciousness. Frontiers in Human Neuroscience, 12, 162. https://doi.org/10.3389/fnhum.2018.00162

[76] Nunez, P.L. and Srinivasan, R. (1981) Electric Fields of the Brain: The Neurophysics of EEG. Oxford University Press, Oxford.

[77] Plant, L.D., Kemp, P.J., Peers, C., Henderson, Z. and Pearson, H.A. (2002) Hypoxic Depolarization of Cerebellar Granule Neurons by Specific Inhibition of TASK-1. Stroke, 33, 2324-2328. https://doi.org/10.1161/01.STR.0000027440.68031.B0

[78] Hynd, M.R., Scott, H.L. and Dodd, P.R. (2004) Glutamate-Mediated Excitotoxicity and Neurodegeneration in Alzheimer's Disease. Neurochemistry International, 45, 583-595. https://doi.org/10.1016/j.neuint.2004.03.007

[79] Rahn, K.A., Slusher, B.S. and Kaplin, A.I. (2012) Glutamate in CNS Neurodegeneration and Cognition and Its Regulation by GCPII Inhibition. Current Medicinal Chemistry, 19, 1335-1345. https://doi.org/10.2174/092986712799462649

[80] Nilsson, G.E. and Lutz, P.L. (2004) Anoxia Tolerant Brains. Journal of Cerebral Blood Flow \& Metabolism, 24, 475-486. https://doi.org/10.1097/00004647-200405000-00001

[81] Bromfield, E.B., Cavazos, J.E. and Sirven, J.I. (2006) Chapter 1, Basic Mechanisms Underlying Seizures and Epilepsy. In: An Introduction to Epilepsy, American Epilepsy Society, West Hartford.

[82] Gao, R. and Penzes, P. (2015) Common Mechanisms of Excitatory and Inhibitory Imbalance in Schizophrenia and Autism Spectrum Disorders. Current Molecular Medicine, 15, 146-167. https://doi.org/10.2174/1566524015666150303003028

[83] Błaszczyk, J.W. (2016) Parkinson’s Disease and Neurodegeneration: GABA-Collapse Hypothesis. Frontiers in Neuroscience, 10, 269. https://doi.org/10.3389/fnins.2016.00269

[84] Levitt, E.S., Abdala, A.P., Paton, J.F.R., Bissonnette, J.M. and Williams, J.T. (2015) $\mu$ Opioid Receptor Activation Hyperpolarizes Respiratory-Controlling Kölliker-Fuse Neurons and Suppresses Post-Inspiratory Drive. The Journal of Physiology, 593, 4453-4469. https://doi.org/10.1113/JP270822

[85] Xue, M., Atallah, B.V. and Scanziani, M. (2014) Equalizing Excitation-Inhibition Ratios across Visual Cortical Neurons. Nature, 511, 596-600.

https://doi.org/10.1038/nature13321

[86] Ren, D. (2011) Sodium Leak Channels in Neuronal Excitability and Rhythmic Behaviors. Neuron, 72, 899-911. https://doi.org/10.1016/j.neuron.2011.12.007

[87] Jerath, R., Crawford, M.W., Barnes, V.A. and Harden, K. (2015) Widespread Depolarization during Expiration: A Source of Respiratory Drive? Medical Hypotheses, 84, 31-37. https://doi.org/10.1016/j.mehy.2014.11.010

[88] Alberts, B., Johnson, A. and Lewis, J. (2002a) Ion Channels and the Electrical Properties of Membranes. In: Molecular Biology of the Cell, Garland Science, New York.

[89] Lambert, N., Chen, Y.-N., Cheng, Y.-C., Li, C.-M., Chen, G.-Y. and Nori, F. (2012) Quantum Biology. Nature Physics, 9, 10. https://doi.org/10.1038/nphys2474

[90] Serway, R., Vuille, C. and Faughn, J. (2008) College Physics. Brooks/Cole, Belmont. 
[91] Gray, H.B. and Winkler, J.R. (2004) Electron Tunneling through Proteins. Quarterly Reviews of Biophysics, 36, 341-372. https://doi.org/10.1017/S0033583503003913

[92] Nagel, Z.D. and Klinman, J.P. (2006) Tunneling and Dynamics in Enzymatic Hydride Transfer. Chemical Reviews, 106, 3095-3118.

https://doi.org/10.1021/cr050301x

[93] Jerath, R., Cearley, S.M., Barnes, V.A. and Junca, S. (2017) The Dynamic Role of Breathing and Cellular Membrane Potentials in the Experience of Consciousness. World Journal of Neuroscience, 7, 66-81. https://doi.org/10.4236/wjns.2017.71007

[94] Rich, P.R. (2003) The Molecular Machinery of Keilin's Respiratory Chain. Biochemical Society Transactions, 31, 1095-1105. https://doi.org/10.1042/bst0311095

[95] Suki, B., Stamenovic, D. and Hubmayr, R. (2011) Lung Parenchymal Mechanics. Comprehensive Physiology, 1, 1317-1351. https://doi.org/10.1002/cphy.c100033

[96] Hogan, C.M. (2011) Respiration. In: Mcginley, M. and Cleveland, C.J., Eds., Encyclopedia of Earth, National Council for Science and the Environment, Washington DC.

[97] Lemasters, J.J. and Hackenbrock, C.R. (1976) Continuous Measurement and Rapid Kinetics of ATP Synthesis in Rat Liver Mitochondria, Mitoplasts and Inner Membrane Vesicles Determined by Firefly-Luciferase Luminescence. European Journal of Biochemistry, 67, 1-10. https://doi.org/10.1111/j.1432-1033.1976.tb10625.x

[98] Alberts, B., Johnson, A. and Lewis, J. (2002b) The Mitochondrion. In: Molecular Biology of the Cell, 4th Edition, Garland Science, New York.

[99] Murray, R.K., Granner, D.K., Mayes, P.A. and Rodwell, V.W. (2003) Harper's Illustrated Biochemistry. Lange Medical Books/ McGraw Hill, New York.

[100] Saroff, H.A. (2007) The Nernst Equation Applied to Oxidation-Reduction Reactions in Myoglobin and Hemoglobin. Evaluation of the Parameters. Biopolymers, 85, 450-455. https://doi.org/10.1002/bip.20652

[101] Bonaventura, C., Henkens, R., Alayash, A.I., Banerjee, S. and Crumbliss, A.L. (2013) Molecular Controls of the Oxygenation and Redox Reactions of Hemoglobin. Antioxidants \& Redox Signaling, 18, 2298-2313. https://doi.org/10.1089/ars.2012.4947

[102] Fogiel, M. (2004) Biology Problem Solver. Research \& Education Association, Piscataway.

[103] Taussig, L. (2008) Chapter 14 Gas Exchange and Acid-Base Physiology. In: Landau, L.I., Ed., Pediatric Respiratory Medicine, 2nd Edition, Mosby, Philadelphia, 179-200.

[104] Reviews, C. (2016) Neuroscience, Exploring the Brain: Psychology, Human Development.

[105] Mortola, J.P. Resting Breathing Pattern and Its Peripheral Modulation. Topics in Respiratory Physiology for Undergraduate Students. https://www.medicine.mcgill.ca/physio/resp-web/TEXT4.htm

[106] Gattinoni, M.D.L., Pesenti, W.D.A., Bombino, M.D.M., Baglioni, M.D.S., Rivolta, M.D.M., Rossi, M.D.F., Rossi, M.D.G., Fumagalli, M.D.R., Marcolin, M.D.R., Mascheroni, M.D.D. and Torresin, P.D.A. (1988) Relationships between Lung Computed Tomographic Density, Gas Exchange, and PEEP in Acute Respiratory Failure. Anesthesiology, 69, 824-832. https://doi.org/10.1097/00000542-198812000-00005 